$(\mathrm{p}<0.05)$ and mitochondrial respiration assessed by a Seahorse flux analyser decreased by $25 \%(\mathrm{p}<0.05)$.

To determine the effects of mtDNA damage, we studied arterial ageing in mice that overexpressed the mitochondrial helicase Twinkle $\left(\mathrm{Tw}^{+}\right)$, or with a mutation in the proof-reading ability of the mitochondrial polymerase gamma (PolG). Twinkle expression restored mtDNA copy number with concurrent improvement in mitochondrial respiration. Twinkle expression delayed all physiological parameters of vascular ageing, associated with decreased collagen and elastin breaks $(\mathrm{p}<0.05)$. In contrast, PolG mice with increased mtDNA damage showed accelerated vascular ageing compared to controls $(\mathrm{p}<0.05)$.

Conclusions We have identified multiple, reproducible parameters of arterial ageing in mice that are detected at far earlier time points than previously described; in particular, compliance, distensibility and $\square$ SI at $44 \mathrm{wk}$ provide the earliest discrimination. Arterial mitochondrial function reduces markedly with age, and accelerates vascular ageing, whereas augmenting mitochondrial function delays ageing, identifying prevention of mtDNA damage and dysfunction as a therapeutic target in ageing.

\section{HYPERPOLARIZED MAGNETIC RESONANCE IMAGING OF CARDIAC INFLAMMATION AND REPAIR}

${ }^{1}$ Andrew JM Lewis* ${ }^{3,1}$ lack J Miller, ${ }^{2,3}$ Oliver J Rider, ${ }^{3}$ Robin P Choudhury, ${ }^{3}$ Stefan Neubauer, ${ }^{1}$ Carolyn A Carr, ${ }^{1}$ Damian J Tyler. 'Department of Physiology, Anatomy and Genetics, University of Oxford, UK; ${ }^{2}$ Department of Physics, Clarendon Laboratory, University of Oxford, UK; ${ }^{3}$ Radcliffe Department of Medicine, University of Oxford, UK

10.1136/heartjnl-2017-311726.234

Myocardial infarction (MI) remains a major killer despite highly optimised systems for the delivery of primary percutaneous coronary intervention (PPCI), highlighting a need for novel therapeutics that could be administered in the days following the event. The healing myocardium undergoes a macrophage driven inflammatory response which is a compelling therapeutic target, though clinical exploration of this process has been limited because conventional imaging techniques cannot assess cellular inflammation in the heart.

We hypothesised that the huge increases in signal-to-noise ratio provided by hyperpolarized MRI could provide a solution to this problem. In rodent models, hyperpolarized [113C]pyruvate MRI using a custom designed metabolite mapping sequence in vivo demonstrated that experimental MI caused intense [1-13C]lactate signal in healing myocardial segments at both day 3 (paralleling the maximal 'inflammatory' phase of the macrophage response) and also at day 7 ('reparative' phase), compared to sham operated controls. Monocyte/ macrophage depletion using clodronate liposomes normalised the [1-13C]lactate signal at both timepoints.

Gene expression analysis of monocytes/macrophages sorted from infarct tissue demonstrated regulation of key enzymes of glycolysis, suggesting that monocyte/macrophage recruitment and metabolic reprogramming of those cells underlies the high lactate signal detected. Hyperpolarized [1-13C]pyruvate MR spectroscopy in macrophage-like cell suspensions confirmed that cellular activation and polarisation almost doubles hyperpolarized lactate label flux rates in vitro; blockade of glycolysis with 2-deoxyglucose (2-DG) in activated macrophage-like cells normalised lactate label flux rates and also markedly inhibited production of key pro-inflammatory cytokines at both mRNA and protein level, without major cytotoxicity.

Systemic administration of 2-deoxyglucose following rodent MI normalised hyperpolarized [1-13C]lactate signal in healing myocardial segments and also caused dose dependent improvement in IL-1 $\beta$ expression in infarct tissue, providing proof-ofconcept of 'MR visible' immunomodulation. Furthermore, cine MRI demonstrated improvements in myocardial remodelling and systolic function in 2-DG treated rats at 3 months. Finally, we present initial human experience of cardiac hyperpolarized [1-13C]pyruvate MR, demonstrating unprecedented improvements in signal-to-noise ratio and highlighting the potential for rapid clinical translation of these findings.

We conclude that hyperpolarized MRI provides a novel biomarker of cardiac inflammation and repair post-MI by detecting the induction of an immuno-metabolic pathway in cardiac macrophages which controls key inflammatory cytokine production and influences myocardial remodelling. In addition to a role in the development of novel therapeutics to improve remodelling post MI, hyperpolarized MRI may have broad applications in other inflammatory cardiovascular diseases.

\section{ATHEROSCLEROTIC INFLAMMATION IMAGING USING ${ }^{68}$ GA-DOTATATE PET VS. ${ }^{18}$ F-FDG PET: A PROSPECTIVE CLINICAL SUDY WITH MOLECULAR AND HISTOLOGICAL VALIDATION}

${ }^{1}$ Jason M Tarkin*, ${ }^{2}$ Francis R Joshi, ${ }^{3}$ Nicholas R Evans, ${ }^{4}$ Mohammed M Chowdhury, ${ }^{1}$ Nichola L Figg, ${ }^{1}$ Aarti V Shah, 'Lakshi T Starks, ${ }^{1}$ Abel Martin-Garrido, ${ }^{5}$ Roido Manavaki, ${ }^{1}$ Emma Yu, ${ }^{6}$ Rhoda E Kuc, ${ }^{7}$ Luigi Grassi, ${ }^{7}$ Roman Kreuzhuber, ${ }^{7}$ Myrto A Kostadima, ${ }^{7}$ Mattia Frontini, ${ }^{8}$ Peter J Kirkpatrick, ${ }^{4}$ Patrick A Coughlin, ${ }^{4,9}$ Deepa Gopalan, ${ }^{3}$ Tim D Fryer, ${ }^{9}$ John R Buscombe, ${ }^{10}$ Ashley M Groves, ${ }^{7,11}$ Willem H Ouwehand, ${ }^{1}$ Martin R Bennett, ${ }^{3}$ Elizabeth A Warburton, ${ }^{6}$ Anthony P Davenport, ${ }^{1}$ James HF Rudd. ${ }^{1}$ Division of Cardiovascular Medicine, University of Cambridge, UK; ${ }^{2}$ Heart Centre, Rigshospitalet, Copenhagen, Denmark; ${ }^{3}$ Department of Clinical Neurosciences, University of Cambridge, UK; ${ }^{4}$ Department of Vascular and Endovascular Surgery, Addenbrooke's Hospital, UK; ${ }^{5}$ Department of Radiology, University of Cambridge, UK; ${ }^{6}$ Experimental Medicine and Immunotherapeutics, University of Cambridge, UK; ' Department of Haematology, University of Cambridge, and NHS Blood and Transport, Cambridge, UK; ${ }^{8}$ Division of Neurosurgery, Addenbrooke's Hospital, UK; ${ }^{9}$ Department of Radiology, Hammersmith Hospital, UK; ${ }^{10}$ Department of Nuclear Medicine, Addenbrooke's Hospital, UK; ${ }^{11}$ Institute of Nuclear Medicine, University College London, UK; ${ }^{12}$ Department of Human Genetics, Wellcome Trust Sanger Institute, Wellcome Trust Genome Campus, UK

\subsection{6/heartjnl-2017-311726.235}

Background Inflammation drives atherosclerotic plaque rupture underlying most clinical events. While inflammation can be measured using 18F-fluorodeoxyglucose (FDG) positron emission tomography (PET), 18F-FDG lacks cell-specificity and is unreliable for coronary imaging owing to myocardial signal spillover. Up-regulation of somatostatin receptor-2 (SST2) occurs in activated macrophages offering a novel inflammation imaging target. 
Methods We comprehensively evaluated 68Ga-DOTATATE, a SST2 PET ligand, for imaging atherosclerosis. Target SSTR2 gene expression in macrophages and other immune cells were tested using population-based RNA-sequencing data. Patients with atherosclerosis $(n=42)$ underwent 68Ga-DOTATATE PET imaging in a prospective head-to-head comparison with $18 \mathrm{~F}$ FDG. 68Ga-DOTATATE autoradiography, immunostaining and quantitative PCR were performed in macrophages and excised carotid specimens from patients who underwent PET imaging. Results Target SSTR2 expression occurred exclusively in "proinflammatory" M1 macrophages, and no other cell type studied in vitro. In clinical imaging, 68Ga-DOTATATE mean of maximum tissue-to-blood ratios (mTBRmax) correctly identified culprit vs. non-culprit arteries in patients with acute coronary syndrome (median difference 0.69 [IQR 0.22 to 1.15 ], $\mathrm{p}=0.008$ ) and transient ischaemic attack or stroke (median difference 0.13 [IQR 0.07 to 0.32 ], $\mathrm{p}=0.003$ ). 68Ga-DOTATATE mTBRmax accurately predicted stable non-culprit coronary lesions with high-risk CT features (ROC AUC 0.86 [95\% CI 0.80 to 0.92], $\mathrm{p}<0.0001$ ), and correlated with Framingham risk score $(\mathrm{r}=0.53$ [95\% $\quad$ CI 0.32 to 0.69 ], $\mathrm{p}<0.0001)$ and vascular inflammation defined by $18 \mathrm{~F}-\mathrm{FDG}$ ( $\mathrm{r}=0.73$ [95\% CI 0.64 to 0.81 ], $\mathrm{p}<0.0001)$. While 18F-FDG mTBRmax also differentiated culprit from non-culprit carotid lesions (median difference 0.12 [IQR 0.0 to 0.23 ], $\mathrm{p}=0.008$ ) and high-risk from lower-risk coronary lesions (ROC AUC 0.76 [ $95 \%$ CI 0.62 to 0.91 ], $\mathrm{p}=0.002$ ), myocardial $18 \mathrm{~F}-\mathrm{FDG}$ spillover rendered coronary $18 \mathrm{~F}-\mathrm{FDG}$ scans uninterpretable in 27 (64\%) patients. In contrast, low myocardial 68Ga-DOTATATE binding allowed unimpeded coronary signal interpretation in all patients without the need for pre-scan fasting. Moreover, histological analysis confirmed specific binding of 68Ga-DOTATATE to SST2 receptors expressed by CD68-positive macrophages in excised carotid plaques. Carotid SSTR2 mRNA was highly correlated with both CD68 mRNA ( $\mathrm{r}=0.93$ [95\% CI 0.49 to 0.99 ]; $\mathrm{p}=0.007$ ) and in vivo 68Ga-DOTATATE PET signals measured from clinical images $(r=0.89$ [95\% CI 0.28 to 0.99], $\mathrm{p}=0.02$ ).

Conclusion We provide gene, cell, plaque and patient-level data, demonstrating that SST2 PET imaging using $68 \mathrm{Ga}-$ DOTATATE represents a macrophage-specific marker of atherosclerotic inflammation that outperforms 18F-FDG in the coronary arteries. Future research will explore the utility of 68Ga-DOTATATE inflammation imaging to classify high-risk patients for aggressive therapeutic intervention.

\section{E EOSINOPHILS HAVE AN ESSENTIAL ROLE IN CARDIAC REPAIR FOLLOWING MYOCARDIAL INFARCTION}

${ }^{1}$ Iqbal S Toor*, ${ }^{2}$ Dominik Rückerl, ${ }^{1}$ Adrian Thomson, ${ }^{3}$ Kare Tang, ${ }^{1}$ David E Newby, ${ }^{4}$ Adriano G Rossi, ${ }^{2}$ Judith E Allen, 'Gillian A Grey. 'BHF/University Centre for Cardiovascular Science, Queen's Medical Research Institute, University of Edinburgh, Edinburgh, UK; ${ }^{2}$ Faculty of Biology, Medicine and Health, School of Biological Sciences, University of Manchester, Manchester, UK; ${ }^{3}$ Essex Cardiothoracic Centre, Basildon and Thurrock Hospitals NHS Foundation Trust, London, UK; ${ }^{4}$ MRC Centre for Inflammation Research, Queen's Medical Research Institute, University of Edinburgh, Edinburgh, UK

\subsection{6/heartjnl-2017-311726.236}

Background Low peripheral blood eosinophil count is associated with increased risk of mortality in ischaemic heart disease patients. Eosinophils contain preformed IL-4 within their cytoplasmic granules, which promotes an anti-inflammatory response and is associated with tissue repair. Whether eosinophils are recruited to the infarct zone and have a role in regulating infarct repair is currently unknown.

Purpose This study sought to investigate the role of eosinophils in infarct healing.

Methods MI was induced by permanent coronary artery ligation in 12-15 week-old male wild-type (WT) BALB/c and eosinophil deficient $\Delta$ dblGATA mice. Cardiac function was assessed 7 days later by high-resolution ultrasound. A cohort of 732 patients undergoing emergency percutaneous coronary intervention for ST-elevation myocardial infarction (STEMI) were followed up for 6 month all-cause mortality.

Results Histochemical staining (Siglec $\mathrm{F}+$ ) and single cell digestion of infarcted WT $\mathrm{BALB} / \mathrm{c}$ hearts revealed significant recruitment of $(\mathrm{CD} 11 \mathrm{~b}+\operatorname{SiglecF}+\mathrm{Ly} 6 \mathrm{Gint})$ eosinophils to the infarcted heart. Genetic eosinophil deficiency in $\triangle$ dblGATA mice led to greater left ventricular dilatation relative to WT $\mathrm{BALB} / \mathrm{c}$ mice and worse cardiac function at Day 7 post-MI. Patients with a low eosinophil count at Day 1 following STEMI had an increased risk of 6 month all-cause mortality. On multivariate analysis the hazard ratio of all-cause mortality in the first tertile of peripheral blood eosinophil count at Day 1 post-MI was 6.97 [2.18-22.32] compared to the highest tertile $(p=0.001)$. Treatment with IL-4 complexes was able to rescue the adverse cardiac remodelling of eosinophil-deficient $\Delta$ dblGATA mice. Expression of plod2, lox and Fmod genes, which are involved in post-translational collagen modification, were increased in hearts from $\triangle$ dblGATA mice. This was accompanied with longer infarct length in $\triangle$ dblGATA mice compared to WT BALB/c mice $(\mathrm{p}=0.01)$.

Conclusions This study provides the first evidence for an essential role of eosinophils in the tissue repair response following MI, through regulating genes associated with connective tissue biogenesis. Patients with a low eosinophil post-MI may benefit from IL-4 therapy to improve outcome. 